\title{
Staggered and extreme localization of electron states in fractal space
}

\author{
Biplab Pa: and Arunava Chakrabarti \\ Department of Physics, University of Kalyani, Kalyani, West Bengal - 741 235, India
}

\begin{abstract}
We present exact analytical results revealing the existence of a countable infinity of unusual single particle states, which are localized with a multitude of localization lengths in a Vicsek fractal network with diamond shaped loops as the 'unit cells'. The family of localized states form clusters of increasing size, much in the sense of Aharonov-Bohm cages [J. Vidal et al., Phys. Rev. Lett. 81, 5888 (1998)], but now without a magnetic field. The length scale at which the localization effect for each of these states sets in can be uniquely predicted following a well defined prescription developed within the framework of real space renormalization group. The scheme allows an exact evaluation of the energy eigenvalue for every such state which is ensured to remain in the spectrum of the system even in the thermodynamic limit. In addition, we discuss the existence of a perfectly conducting state at the band center of this geometry and the influence of a uniform magnetic field threading each elementary plaquette of the lattice on its spectral properties. Of particular interest is the case of extreme localization of single particle states when the magnetic flux equals half the fundamental flux quantum.
\end{abstract}

PACS numbers: $73.20 . \mathrm{Fz}, 73.22 . \mathrm{Dj}$

\section{INTRODUCTION}

Interplay of lattice topology and quantum interference effects is known to give rise to exotic electronic spectrum in solid systems that has been studied in details over several decades by now. The subject is still being pursued with vigor and with an aim to achieve comprehensive control over coherent transport in low dimensions.

In quantum interference and related transport mechanism a pivotal role is played by the localization of electronic eigenstates in presence of disorder. Such localization, known as the Anderson localization [1] upholds a central result that, in one dimension with arbitrary disorder, all the single particle states will be exponentially localized, and the same was shown to ring true in two dimensions as well [2]. Since then, extensive research has been undertaken to understand the fundamentals of localization effects, studies ranging from electronic states in random lattice models [3]- 8], to the Anderson localization of light 9,10 , spin freezing in one dimensional semiconductors [11], and localization in optical lattices [12], to name a few. Matter waves can also be localized in deterministic potentials sharing certain features of random disorder [13, 14]. Recent experiments reveal the Anderson localization of non-interacting Bose-Einstein condensates in one dimensional matter waveguides, where the random potential has been generated by laser speckles [15]. Similar experiments have also been reported to study the Anderson localization in optical lattices [16, 17], and in the cases of microwaves [18] and of classical waves in weakly disordered one dimensional stack of meta-materials [19].

Variations of the classic Anderson localization are also well known by now. Isolated de-localized (extended) single particle states exist, even in a disordered one di-

\footnotetext{
*E-mail: biplabpal@klyuniv.ac.in
}

mensional chain of atomic potentials, resulting out of a kind of spatial correlation [20, 21], in 1-d quasiperiodic chains [22]-[26], or, in certain kinds of deterministic fractal geometries [27]-29]. Crossover from an insulating to a metallic spectral behavior in correlated disordered two-legged ladder networks have also been reported recently [30, 31].

A curious point, apparently gone unnoticed or unappreciated so far is that, while a precise determination of the eigenvalues corresponding to the extended single particle states is possible in the above cases of correlated and deterministic disorder, the task seems to be practically impossible when it comes to an exact evaluation of eigenvalues of the localized states in a random or even a deterministically disordered system in the thermodynamic limit. It should be appreciated that, though a direct diagonalization of the Hamiltonian for a finite size of the system yields eigenvalues of the localized states (for a disordered or a deterministically disordered system), there is no apriory reason to assume that these eigenvalues remain in the spectrum when the system grows in size, and tends to infinity. In fact, for a deterministic fractal geometry that offers a singular continuous spectrum, it is almost impossible to hit the exact eigenvalues corresponding to the states that will finally be localized on an infinite lattice. To the best of our knowledge, this issue remains unaddressed so far in the literature.

Can one really identify the localized states and extract the corresponding eigenvalues for a deterministically disordered system? In the present communication we address ourselves this question, and take up the task of critically examining the spectral properties of a Vicsek fractal network 32] consisting of diamond shaped loops within a tight binding formalism. While looking for the localized state eigenvalues and the nature of localization are indeed the major factors driving this work, other interests in such a study are related to the general spectral character and magneto-transport in such systems. The 
motivation behind the latter part of the work may be summarised as follows. A diamond-Vicsek network (see Fig. 1) provides an interesting geometry in which the 'open' character of a typical Vicsek pattern is preserved along with the presence of closed loops in shorter scales of length. This is in marked contrast to the much studied Sierpinski gasket [33, 34], which is a closed structure, or to the other open tree fractals 35] or even an alternative version of the Vicsek fractal without any local closed loops [36]. The presence of these loops effectively generates a longer ranged interaction between the atomic sites occupying the various vertices, and its effect on the electron localization or de-localization is worth studying.

Secondly, linear arrays of diamond networks have already drawn considerable attention in recent years in the context of charge and spin transport properties [37]-39], being shown to behave as a flux controlled $n$ - or $p$-type semiconductor [37], or as a prospective candidate of an elegant spin filter [38, 39]. The influence of a topological variation in the arrangement of such loops on the spectral properties of the system is thus worth investigating, both from the standpoint of fundamental physics, and from the perspective of device technology. We choose such a deterministic geometry to make an analytical attack on the system possible.

We find extremely interesting results in the context of localization of electronic states. In the absence of any external magnetic field, a countable infinity of localized states can be precisely detected with a multitude of localization lengths. One can work out an exact mathematical prescription to specify the length scale at which the onset of localization takes place. The localization can in principle, be delayed (staggered) in position space and the corresponding energy eigenvalues can be exactly evaluated following the same prescription based on a real space renormalization group (RSRG) method. In addition, it is shown that for a given set of parameters, the center of the spectrum corresponds to a perfectly extended eigenstate, with the parameters of the Hamiltonian exhibiting a fixed point behavior. Switching on a magnetic field opens up gaps in the spectrum in general, and even leads to an extreme localization of all the single particle states in the sense of formation of the Aharonov-Bohm cages [40].

In what follows we describe the results. In section II, the model and the mathematical method of handling the problem are presented. Section III and IV include the results and their analyses related to the spectral properties without and with the magnetic field respectively. The two terminal transport study is carried out in section $\mathrm{V}$ and in section VI we draw our conclusions.

\section{THE SYSTEM AND THE MATHEMATICAL FORMULATION}

\section{A. The Hamiltonian}

We refer to Fig. 1(a) which illustrates the second gen- eration of a Vicsek geometry with diamond shaped loops. As, at one stage we shall be considering the effect of a magnetic field on the spectral properties, we show in the figure the flux distribution. Each plaquette is threaded by a uniform magnetic flux $\Phi$. The atomic sites are assigned different status depending on their positions and neighborhood in the lattice, namely, the sites in the bulk are marked as ' $B$ ', while ' $A$ ' refers to the sites sitting at the edges. The magnetic field breaks the time reversal symmetry along the edges of every diamond. The Hamiltonian, in the tight binding formalism is written as,

$$
\boldsymbol{H}=\sum_{i} \epsilon_{i}|i\rangle\langle i|+\sum_{\langle i j\rangle}\left[t_{i j} e^{i \theta_{i j}}|i\rangle\left\langle j\left|+t_{j i} e^{-i \theta_{i j}}\right| j\right\rangle\langle i|\right]
$$

where, $\epsilon_{i}$ is the on-site potential at the $i$-th site, and has a value $\epsilon_{A}$ or $\epsilon_{B}$ depending on whether its an 'edge' site or a 'bulk' one. The uniform nearest neighbor hopping amplitude is $t_{i j}=t$ along the edges, and $t_{i j}=\lambda$ when $i$ and $j$ refer to the opposite vertices, connected by a diagonal. Thus we keep the provision of including hopping beyond the nearest neighbors. $\theta_{i j}$ is the Peierls' phase [41] given by, $\theta_{i j}=2 \pi \Phi / 4 \Phi_{0}$ for hopping along an edge. $\Phi_{0}=h c / e$ is the fundamental flux quantum. From symmetry considerations, $\theta_{i j}=0$ when the electron hops across a diagonal, that is, when $t_{i j}=\lambda$.

\section{B. The RSRG scheme}

An elegant way of handling such self-similar systems is to use the real space renormalization group (RSRG) method 42] where one can decimate out a subset of atomic sites from the original lattice to get a scaled version of it (Fig. 1(b)). This is easily done by writing down in details the set of difference equations,

$$
\left(E-\epsilon_{i}\right) \psi_{i}=\sum_{j} t_{i j} e^{i \theta_{i j}} \psi_{j}
$$

where, $\psi_{i}$ denotes the amplitude of the wave function at the $i$-th site, and $\theta_{i j}$ is the Peierls' phase associated with the hopping matrix element connecting the $i$-th and the $j$-th sites. We begin with nearest neighbor hopping only (that is, we set $\lambda=0$ at the beginning). However, such a decimation automatically generates the second neighbor hopping across the diagonals of an inflated diamond as shown by the dotted line in Fig. 1(b). The range of interactions of course does not increase beyond this on further renormalization. It is therefore advisable to retain $\lambda$ in the Hamiltonian from the very beginning. One can easily compare the results obtained by switching $\lambda$ on or off. The recursion relations of the on-site potentials and the hopping integrals are provided below.

$$
\begin{aligned}
\epsilon_{A}^{\prime} & =\epsilon_{A}+\left[p t_{f}+p^{*} t_{b}+\alpha \lambda_{1}\right] \\
\epsilon_{B}^{\prime} & =\epsilon_{B}+2\left[p t_{f}+p^{*} t_{b}+\alpha \lambda_{1}\right] \\
t_{f}^{\prime} & =\beta \lambda_{1} ; t_{b}^{\prime}=\beta^{*} \lambda_{1} ; \lambda^{\prime}=\gamma \lambda_{1}
\end{aligned}
$$




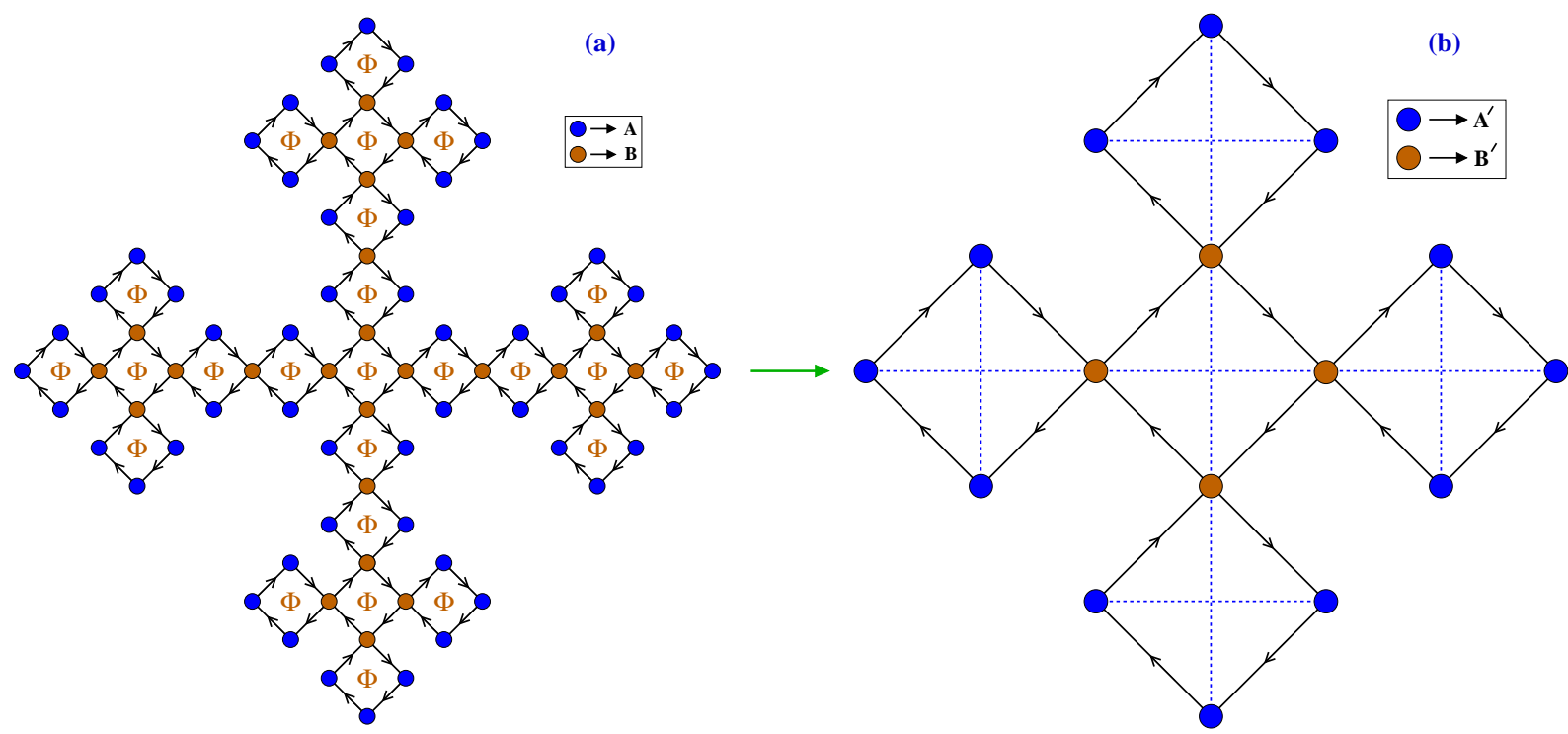

FIG. 1: (Color online) (a) Schematic view of the second generation of an infinite diamond-Vicsek network with each diamond plaquette threaded by a uniform magnetic flux $\Phi$. The edge sites are named ' $A$ ' (blue circles) and the bulk sites are marked as ' $B$ ' (orange circles), and the arrow indicates the direction of the forward hopping. We exclude any second neighbor hopping at the beginning. (b) Renormalized version of (a) with the dotted lines indicating the diagonal hopping which is generated due to renormalization.

where, $\alpha=\left[\left(E-\bar{\epsilon}_{B}\right) \lambda_{1}\right] / \xi_{3} ; \beta=\left[\left(E-\bar{\epsilon}_{B}\right) \bar{t}_{f}+\lambda_{2} \bar{t}_{b}\right] / \xi_{3}$; $\beta^{*}=\left[\left(E-\bar{\epsilon}_{B}\right) \bar{t}_{b}+\lambda_{2} \bar{t}_{f}\right] / \xi_{3} ; \gamma=\lambda_{1} \lambda_{2} / \xi_{3} ; \xi_{3}=(E-$ $\left.\bar{\epsilon}_{B}\right)^{2}-\lambda_{2}^{2}$,

with, $\bar{\epsilon}_{B}=\tilde{\epsilon}_{B}+w^{*} t_{f}+w t_{b} ; \bar{t}_{f}=u t_{f}+v t_{b} ; \bar{t}_{b}=$ $v t_{f}+u t_{b} ; \lambda_{2}=\lambda+w t_{f}+w^{*} t_{b}$.

Here, $u=\left[\left(E-\tilde{\epsilon}_{B}\right) \lambda_{1}\right] / \xi_{2} ; v=\lambda \lambda_{1} / \xi_{2} ; w=[(E-$ $\left.\left.\tilde{\epsilon}_{B}\right) t_{f}+\lambda t_{b}\right] / \xi_{2} ; w^{*}=\left[\left(E-\tilde{\epsilon}_{B}\right) t_{b}+\lambda t_{f}\right] / \xi_{2} ; \xi_{2}=(E-$ $\left.\tilde{\epsilon}_{B}\right)^{2}-\lambda^{2}$,

with, $\tilde{\epsilon}_{B}=\epsilon_{B}+p t_{f}+p^{*} t_{b} ; \lambda_{1}=\lambda+p^{*} t_{f}+p t_{b}$ and $p=\left[\left(E-\epsilon_{A}\right) t_{b}+\lambda t_{f}\right] / \xi_{1} ; p^{*}=\left[\left(E-\epsilon_{A}\right) t_{f}+\lambda t_{b}\right] / \xi_{1} ;$ $\xi_{1}=\left(E-\epsilon_{A}\right)^{2}-\lambda^{2}$.

In the above expression, $t_{f}=t_{b}^{*}=t e^{i \theta}$, where $\theta=$ $2 \pi \Phi / 4 \Phi_{0}$ is the constant Peierls' phase. The above recursion relations are then used to obtain information about the local density of states (LDOS) at specific sites of the system, and the character of the single particle states, as discussed below.

\section{SPECTRAL PROPERTIES WITH ZERO MAGNETIC FIELD}

\section{A. Local density of states in zero magnetic field and with $\lambda=0$}

Using the standard decimation procedure 42], the LDOS at the edge $(A)$ and the bulk $(B)$ sites can easily be obtained through the local Green's functions. For simplicity we present in Fig. 2(2) (a) the LDOS at a $B$-site only, given by,

$$
\rho^{(B)}(E)=\lim _{\eta \rightarrow 0}\left[-\frac{1}{\pi} \operatorname{Im}\left\{G^{(B)}(E+i \eta)\right\}\right]
$$

where, $G^{(B)}(E+i \eta)=\left(E+i \eta-\epsilon_{B}^{*}\right)^{-1}, \epsilon_{B}^{*}$ being the fixed point value of the relevant on-site potential at the $B$-site, obtained by iterating Eq. (3). We have set $\epsilon_{A}=\epsilon_{B}=0$, $t=1$ and $\lambda=0$, and there is no magnetic field (i.e., $\Phi=0$ ). The LDOS shows a dense packing of eigenstates over a finite range of energy centered at $E=0$. We have minutely examined this continuum by fine scanning an energy interval around $E=0$, and show it in Fig. 2(b). The continuum seems to persist. In the neighborhood of the band-center and within the apparent 'continuum', the hopping integral remains non-zero over a substantial number of RSRG iteration steps. The number of such steps $n$ depends on the chosen energy and indicates that the corresponding eigenfunction is either of an extended character, or, at least, has very large localization length. This aspect will be further discussed in the following subsection in relation to the so called staggered localization effect.

A particularly interesting state is the band center, viz., $E=0$, where the entire parameter space $\left\{\epsilon_{A}, \epsilon_{B}, t, \lambda\right\}$ exhibits a one cycle fixed point behavior, viz., $\left\{\epsilon_{A}(n+1), \epsilon_{B}(n+1), t(n+1), \lambda(n+1)\right\}=$ $\left\{\epsilon_{A}(n), \epsilon_{B}(n), t(n), \lambda(n)\right\}$ for $n \geq 1$. $n$ stands for the RSRG iteration step. $\lambda$ at this special energy remains zero throughout the iteration. We conclude that the eigenstate at the band-center is definitely extended, but is of a non-Bloch character. The general behavior of the hopping integrals under successive RSRG iterations is 

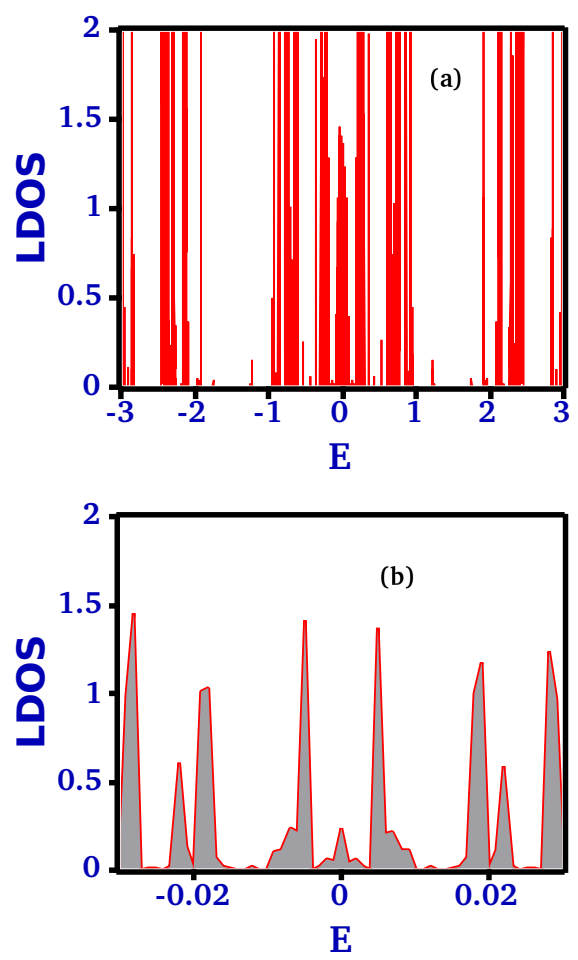

FIG. 2: (Color online) (a) LDOS- $E$ plot at the bulk sites ( $B$-type) of an infinite diamond-Vicsek network in absence of the magnetic field $(\Phi=0)$. The other parameters are $\epsilon_{A}=\epsilon_{B}=0, t=1$ and $\lambda=0$. (b) A highlighted version of (a) around the center $E=0$.

suggestive of the fact that this central extended eigenstate might be flanked on either side by a countable infinity of eigenstates which belong either to the extended category, or have very large localization lengths.

\section{B. Exact construction of eigenstates}

The inherent self-similarity of the deterministic fractals allows for the construction of exact distribution of amplitudes of the eigenstates, by suitably exploiting Eq. (2). Previous attempts in this regard have unfolded extended non-Bloch states (atypically extended states) in the cases of an open loop Vicsek fractal [43] or a closed loop diamond hierarchical geometry 44]. The present lattice offers a richer spectrum, allowing one to explicitly construct localized states extending over clusters of lattice points of various sizes on the parent lattice. The planar extent of such clusters depends on the eigenvalue corresponding to the localized state, and can be small or enormous.

To elaborate, let us consider the solutions of the equation,

$$
E=\epsilon_{B}(n)-2 \lambda(n)
$$

where, $n$ refers to the stage of renormalization. This is in general, a polynomial equation in $E$. The zero's of the polynomial will be eigenvalues of the infinite system if, and only if, with them one can satisfy Eq. (2) locally at every vertex of the lattice, even when the lattice grows infinitely large. This task can be accomplished by trying to draw a non-trivial distribution of amplitudes for an energy obtained from Eq. (5) on the undecimated vertices of an $n$-step renormalized lattice, and then trying to figure out the amplitude distribution on the original lattice at the bare length scale. Let us discuss two specific cases at first.

Case I: We begin with the un-renormalized lattice. Now $n=0$, and with $\epsilon_{A}=\epsilon_{B}=0$ and $\lambda=0$ the solution of Eq. (5) is $E=0$. One can construct an eigenfunction for $E=0$ with amplitudes equal to \pm 1 distributed alternately at the ' $B$ ' sites along the major $X$-and $Y$-axes. The difference equation, viz., Eq. (2) can then easily be satisfied for all other intermediate vertices using the values 0 or \pm 1 .

Case II: The above idea can indeed be extended to higher values of $n$, as we demonstrate in Fig. 3(a) for $n=1$, and discuss below. Let us extract the roots of the Eq. (5) for $n=1$. The roots are $E=0$ and $\pm \sqrt{6}$ for $\epsilon_{A}=\epsilon_{B}=0, t=1$ and $\lambda=0$ initially. We explain the construction of amplitudes for $E=\sqrt{6}$. The trick in this case is to place the values \pm 1 periodically along the major $X$-and $Y$-axes, and to assign an amplitude equal to zero at every 'edge' ( $A$-type site) on a one step renormalized lattice. The amplitudes at the intermediate sites of the original lattice are then systematically evaluated using Eq. (2). We show it in Fig. 3)(a) on a small portion of an infinite lattice. The bigger square boxes with thick, highlighted edges represent the one step renormalized lattice. The construction depicted on a smaller scale can be extended to see that the distribution holds on a lattice of larger spatial extent (Fig. 3(b)). In fact it holds even on a lattice of an arbitrarily large size, where the end sites are not actually visible. As, according to our earlier argument, we are able to satisfy Eq. (2) locally at every vertex while drawing this distribution, $E=\sqrt{6}$ is definitely an eigenvalue of the infinite system, a fact that has been cross-checked by evaluation the LDOS at the $A$ and the $B$-sites at this special energy. We get a stable, finite value of the LDOS which supports our argument above.

Looking back at Fig. 3 the solid black dots represent non-zero values of the amplitude while an open circle represents an amplitude equal to zero. Non-zero amplitudes, represented by solid circles, have the values equal to $\pm 1, \pm \sqrt{6} / 4$ (depicted by the letter $a$ ) and $\pm 1 / 2$ (the letter $b$ ), distributed suitably so as satisfy Eq. (2) consistently at every intermediate vertex on the original lattice. The grey shaded clusters in Fig. 3(a) embrace the nonzero amplitudes only, while every yellow shaded zone is surrounded by vertices where amplitudes are zero. The significant observation is that, clusters of non- zero amplitude span over a finite distance, but ultimately get decoupled from each other on a larger scale of length. 

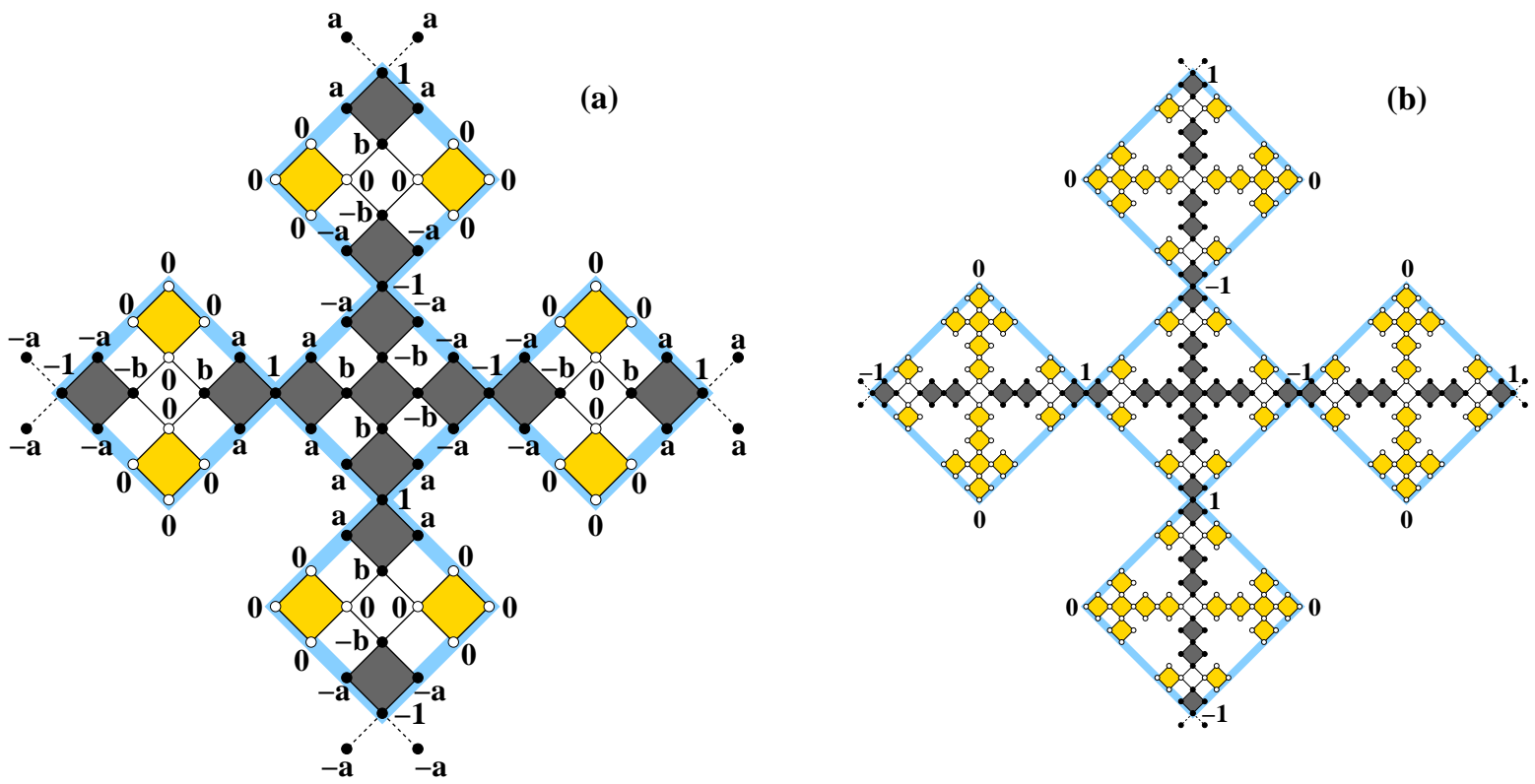

FIG. 3: (Color online) (a) Distribution of amplitudes of the wave functions at $E=\sqrt{6}$ (obtained by solving Eq. (5) for $n=1$ ) on a 2nd generation lattice. The dark shaded plaquettes (grey ones) embrace atomic sites with non-zero amplitudes (black solid circles) and the light shaded plaquettes (yellow ones) are surrounded by atomic sites with zero amplitudes (white empty circles). The initial parameters are chosen as $\Phi=0, \epsilon_{A}=\epsilon_{B}=0, t=1$ and $\lambda=0$. In the figure, $a=\sqrt{6} / 4$ and $b=1 / 2$. (b) Distribution of amplitudes of the wave functions at $E=\sqrt{6}$ on a 3rd generation lattice, the other parameters and symbols are same as (a). In the figures (a) and (b), the highlighted thick (blue) lines represent one-step and two-step renormalized lattice respectively.

This can be appreciated if we look at Fig. 3(b) which is a larger version of the previous figure. The grey shaded clusters are distributed along the principal $X$ - and $Y$ axes, but are decoupled from each other beyond a certain extent by the unfilled white boxes. The yellow clusters representing amplitude-voids are now seen to span larger spatial distances. A similar construction is possible for $E=-\sqrt{6}$ which is another solution of Eq. (5) for $n=1$.

\section{Staggered localization}

It is appearent from the above discussion that the eigenfunction corresponding to $E= \pm \sqrt{6}$ will be localized in the fractal space, as the spanning clusters of different sizes and embracing non-zero amplitudes ultimately get decoupled from one another. This is easily re-confirmed by studying the evolution of the hopping integrals under successive RSRG steps. The hopping integrals $t$ and $\lambda$ (zero initially, but grows later) remain non-zero at the first stage of RSRG (that is, $n=1$ ), indicating that the nearest neighboring sites on a one step renormalized lattice will have a non-zero overlap of the eigenfunctions. They start decaying for $n \geq 2$ with the decay in $\lambda(n)$ taking place at a much slower rate compared to $t(n)$. This indicates that over larger scale of length the corresponding states are ultimately localized, but the effect is a weak one.
This observation immediately leads to an innovative way of exactly determining a localized eigenstate on such a deterministic geometry. It should be appreciated that though it is not unnatural that most of the single particle states will be Anderson-localized in the absence of any translational order, nevertheless an exact prescription of the determination of any localized eigenvalue is not easy to obtain, and has not been reported so far in the literature, to the best of our knowledge. We do it using the following method.

We can solve Eq. (5) in principle, for any $n$. For example, we have done it explicitly for $n=1, n=2, n=3$ and $n=4$. With the same set of parameters as discussed above, the roots of Eq. (5) for $n=2$ are, $E=0$, $\pm \sqrt{6}, \pm 2.11619, \pm 0.77508, \pm 2.98681$. As we observe, the roots for the $n=1$ stage, viz., $E=0$ and $\pm \sqrt{6}$ are included in this set for $n=2$. $E=0$ corresponds to the extended state and $E= \pm \sqrt{6}$ provide two localized states we already know. For each of the additional roots, viz., $E= \pm 2.11619, \pm 0.77508, \pm 2.98681$, the hopping integrals $t$ and $\lambda$ remain non-zero (with considerable magnitude) up to the second stage of iteration $(n=2)$, and starts to lose their 'strengths' as the renormalization progresses. Finally, for large ' $n$ ' the hopping integrals become zero.

The above observation implies that, using a subset of energy values extracted at the stage $n=2(E=$ $\pm 2.11619, \pm 0.77508$ and \pm 2.98681$)$, we can work out 
eigenfunctions which will span bigger clusters of lattice points on the original lattice compared to those obtained from $n=1$. The states will appear to be 'extended' when viewed on a finite diamond-Vicsek fractal at the second generation, but will eventually be localized on a lattice in the thermodynamic limit. In Fig. 4 we show the dis-

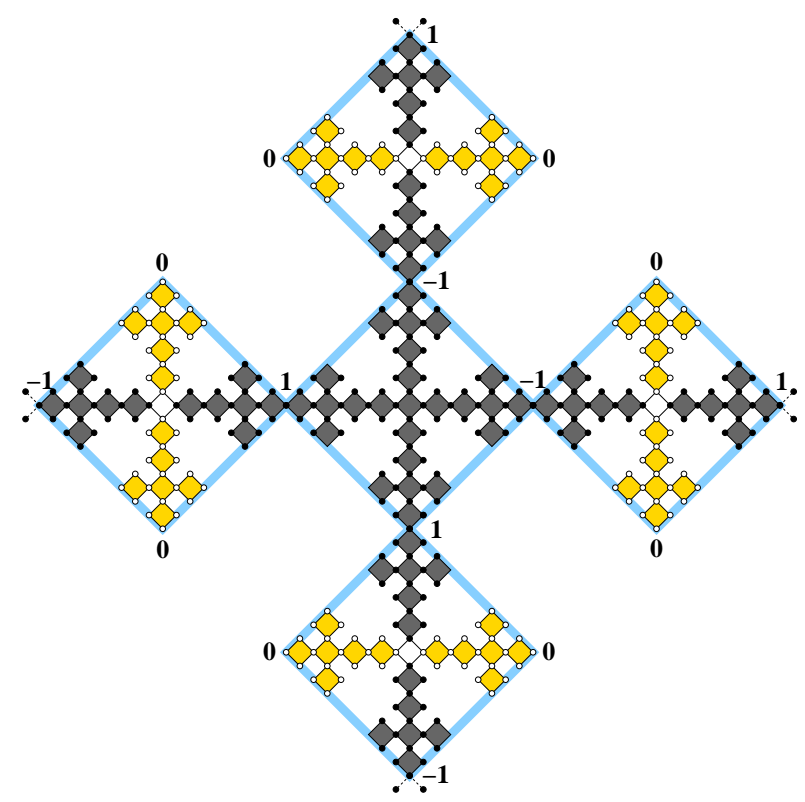

FIG. 4: (Color online) Distribution of amplitudes of the wave functions at $E=0.77508$ (obtained by solving Eq. (5) for $n=2$ ) on a 3rd generation lattice. The other parameters and symbols are same as FIG. 3 The thick, highlighting blue lines represent a two-step renormalized lattice.

tribution of amplitudes for $E=0.77508$, a value that is obtained from Eq. (5) for $n=2$. The enlargement in the cluster-size having non-zero values of the amplitude in comparison to the $n=1$ case (Fig. 3 (b)) is obvious. The spanning clusters finally get decoupled from each other, just as it was for the $n=1$ case. But, now this decoupling occurs at a larger length scale.

It is now easy to foresee what is going to happen for $n=3,4$ and beyond. For any $n=\ell$ we will be getting roots of Eq. (5), subsets of which are solutions of Eq. (5) for $n=1,2, \ldots, \ell-1$. For these subsets, the decay in the hopping integrals will begin at $n>1, n>2, \ldots$, $n>\ell-1$. For the roots in addition to these, the hopping integrals lose their strengths and finally decay, from $n>\ell$. Thus, the latter eigenvalues will correspond to localized eigenstates, the localization being delayed (staggered) in space with localization lengths much larger than the previous ones. When mapped back on to the original lattice, the amplitudes for these additional roots will be found to span clusters of increasing size. The exact size of the spanning clusters will be determined by the value of $n$.

The roots of Eq. (5) are found to cluster around the value $E=0$ symmetrically, and tend to densely fill the neighborhood of $E=0$, at which the single extended eigenstate determined so far resides. The clustering of the eigenvalues is shown in Fig. 5. This dense filling of the

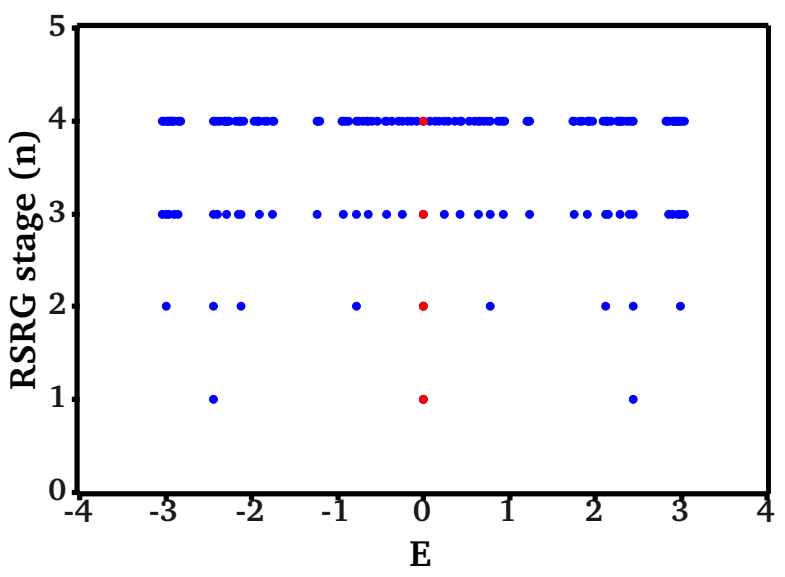

FIG. 5: (Color online) Distribution of energy eigenvalues $E$ obtained from the Eq. (5) for different RSRG stage $n$. The central dot (red one) at $E=0$ represents the eigenvalue for the extended eigenstate.

eigenvalue spectrum around the center is also reflected in the apparent continuum observed in the density of states (Fig. 2(b)).

\section{SPECTRAL PROPERTIES WITH NON-ZERO MAGNETIC FIELD}

A. The energy eigenvalue spectrum

We have obtained the energy eigenvalue distribution (Fig. 6) as a function of the magnetic flux $\Phi$ enclosed in

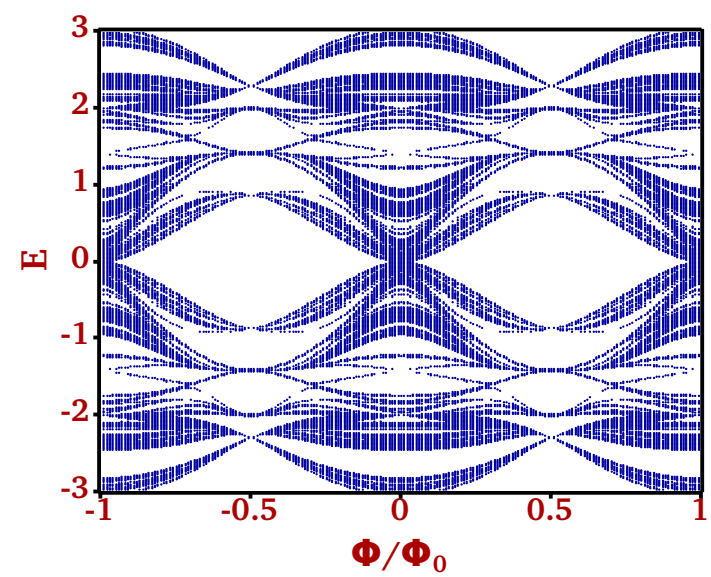

FIG. 6: (Color online) Energy eigenvalue spectrum of an infinite diamond- Vicsek fractal as a function the magnetic flux $\Phi$. We have chosen $\epsilon_{A}=\epsilon_{B}=0, t=1$ and $\lambda=0$.

each basic plaquette for an infinite size diamond-Vicsek fractal. We have examined the formation of the bands 
and the gaps with the variation of magnetic flux $\Phi$. To obtain the energy spectrum, we have calculated the local density of states (LDOS) at both ' $A$ ' and ' $B$ ' sites by fixing the value of the energy $E$ and varying the magnetic flux $\Phi$ from -1 to 1 , repeated the above process for different values of energy $E$, and picked up those values of energy $E$ and magnetic flux $\Phi$ for which we get a nonzero LDOS either at an $A$-site or a $B$-site. Thus Fig. 6 is representative of an infinite lattice.

In Fig. 6, we can clearly observe the formation of multiple bands and gaps, and how a variation of magnetic flux $\Phi$ leads to band overlapping. The band crossing is maximum at the center (around $\Phi=0$ ) and the density of allowed energy eigenvalues is large is this area. As we shift form $\Phi=0$ on either side, there is thinning of allowed energy eigenvalues. And finally at $\Phi=\Phi_{0} / 2$, only four energy eigenvalues are allowed indicating an extreme localization of the electronic states which is discussed in details in the next subsection. Fig. 6 corroborates this last observation, though due to limit of resolution of the diagram, the four eigenvalues are not clearly seen there. This is resolved in the next diagram (Fig. 7(b)).

\section{B. Extreme localization of the electronic states}

In absence of magnetic field, there was clearly a nonzero value of LDOS at the center of the spectrum (around $E=0$ ) (Fig. 2(a)). As soon as the magnetic field is switched on, a wide gap opens up in the LDOS spectrum around $E=0$ (Fig. [7(a)). The gap becomes wider as we increase the value of magnetic flux $\Phi$, finally leading to an extreme localization of electronic states (Fig. 7(b)) at the half flux quantum (i.e., $\Phi=\Phi_{0} / 2$ ). The four separate lines in Fig. 7 (b) (at $E= \pm \sqrt{2}$ and at $E= \pm 2$ ) correspond to four highly degenerate localized states pinned at the ' $A$ '- and ' $B$ '-sites respectively. This observation is in accordance with Vidal et al.'s result [40], where the Aharonov-Bohm caging of the localized orbitals under the action of an external magnetic field was discussed.

The origin of the four localized state eigenvalues above can be easily explained if one appreciates that at $\Phi=$ $\Phi_{0} / 2$, the effective coupling between the sites at the vertices of an elementary rhombus, viz., $t^{\text {eff }}=\left[2 t^{2} /(E-\right.$ $\left.\left.\epsilon_{A}\right)\right] \cos \left(\pi \Phi / \Phi_{0}\right)$, becomes equal to zero. In that case one is left with only two types of atomic sites, decoupled from each other, and having effective on-site potential energies

$$
\begin{aligned}
& \epsilon_{A}^{e f f}=\epsilon_{A}+\frac{2 t^{2}}{E-\epsilon_{B}} \\
& \epsilon_{B}^{e f f}=\epsilon_{B}+\frac{4 t^{2}}{E-\epsilon_{A}}
\end{aligned}
$$

for the 'edge' and the 'bulk' sites respectively. With $\epsilon_{A}=$
$\epsilon_{B}=0$ and $t=1$, the localized states are obtained by setting $E=\epsilon_{A}^{\text {eff }}$ and $E=\epsilon_{B}^{\text {eff }}$, which yield the values $E= \pm \sqrt{2}$ and \pm 2 respectively. These are the energy
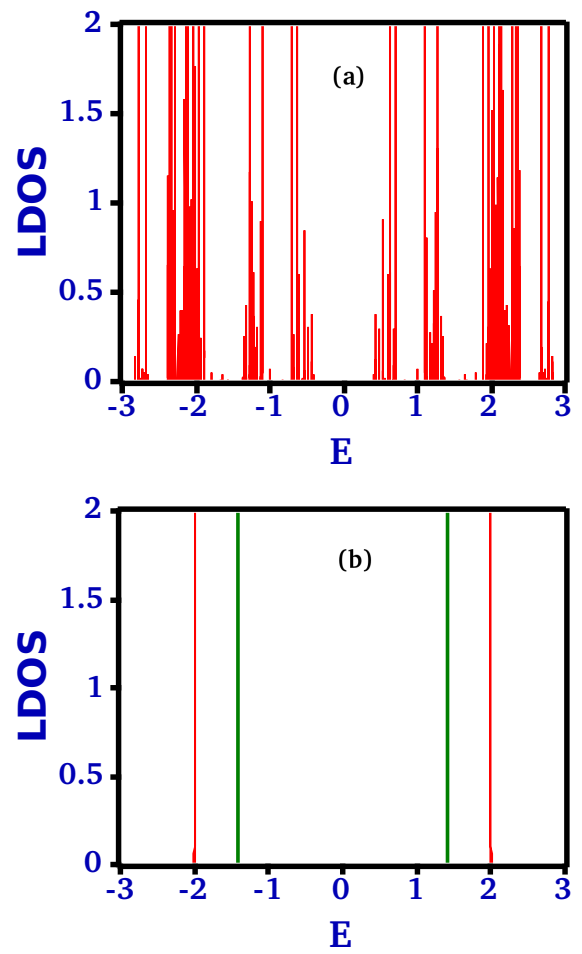

FIG. 7: (Color online) (a) LDOS- $E$ plot at the bulk sites ( $B$ type) of an infinite diamond-Vicsek network with $\Phi=\Phi_{0} / 4$, and (b) is for $\Phi=\Phi_{0} / 2$. The red lines correspond to LDOS at $B$-sites and the green lines correspond to LDOS at $A$-sites. We have chosen $\epsilon_{A}=\epsilon_{B}=0, t=1$ and $\lambda=0$.

eigenvalues at which extreme localization is observed, as shown in Fig. 7(b).

\section{TWO TERMINAL CONDUCTANCE FOR A FINITE LATTICE}

To get the two terminal conductance for a finite size diamond-Vicsek fractal, we attach the system between two semi-infinite one-dimensional ordered metallic leads, namely, the source and the drain. The leads, in the tight binding model, are described by a constant on-site potential $\epsilon_{l}$ and a nearest neighbor hopping integral $t_{l}$. We then successively renormalize the system to reduce it into an effective di-atomic system [4], consisting of two renormalized atoms, each having an effective on-site potential equal to $U$ and with an effective hopping integral $V$ between them. The transmission coefficient across the effective dimer is given by the well known formula [45], 


$$
T=\frac{4 \sin ^{2} k a}{\left[\left(M_{12}-M_{21}\right)+\left(M_{11}-M_{22}\right) \cos k a\right]^{2}+\left[\left(M_{11}+M_{22}\right) \sin k a\right]^{2}}
$$

where, $M_{11}=\frac{(E-U)^{2}}{V t_{l}}-\frac{V}{t_{l}}, M_{12}=-\frac{(E-U)}{V}, M_{21}=$ $-M_{12}, \quad M_{22}=-\frac{t_{l}}{V}$ are the matrix elements of the transfer matrix for the effective di-atomic system, and $\cos k a=\left(E-\epsilon_{l}\right) / 2 t_{l}$, ' $a$ ' being the lattice constant and is taken to be equal to unity throughout the calculation.

In Fig. 8, we have shown the two-terminal transmission characteristics for a 3rd generation system for different values of magnetic flux $\Phi$. For $\Phi=0$, the system exhibits a continuous high transmission window over a region at the center (Fig. 8(a)). This is due to the fact that on ei-
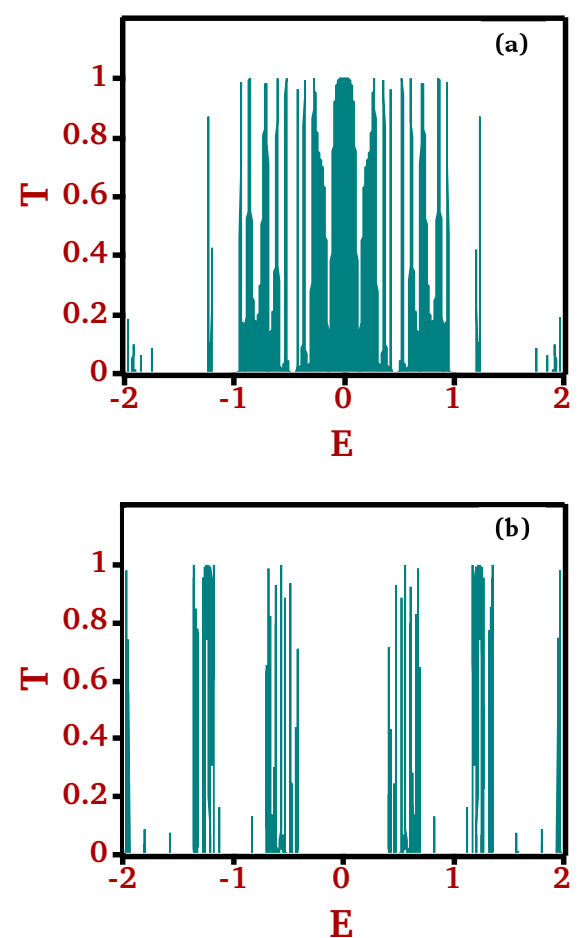

FIG. 8: (Color online) Transmission characteristics for a 3rd generation system. (a) is for $\Phi=0$ and (b) is for $\Phi=\Phi_{0} / 4$. The other parameters are $\epsilon_{A}=\epsilon_{B}=0, t=1$ and $\lambda=0$. The lead parameters are $\epsilon_{l}=0$ and $t_{l}=1$.

ther side of $E=0$, the energy eigenvalues become quite densely packed. The corresponding eigenfunctions have localization lengths extending much beyond the 3rd generation fractal.

As we tune the magnetic flux to a non-zero value, e.g., $\Phi=\Phi_{0} / 4$, the transmittivity of the system drastically decreases (Fig. 8(b)) and with increase in $\Phi$, the value of the transmission coefficient $T$ decreases more and more and finally at $\Phi=\Phi_{0} / 2$, the system becomes completely opaque to an incoming electron. So by fixing the Fermi level of the electron to a particular energy, say at $E=0$, one can easily transform the system from a conducting one to an insulating one by tuning the external magnetic flux $\Phi$ suitably.

\section{CONCLUDING REMARKS}

In conclusion, we have examined the energy spectrum of a Vicsek geometry consisting of diamond shaped loops. The major result is that we have been able to identify a countable infinity of localized eigenstates displaying a multitude of localization lengths. A prescription is given for an exact determination of the eigenvalues corresponding to all such states, a problem that is far from trivial in the case of a deterministically disordered system. The localized states span across the fractal space in clusters of increasing sizes, the size being precisely controlled by the length scale at which the energy eigenvalue is extracted. The onset of localization can be exactly predicted from the stage of RSRG. In addition, the application of a uniform magnetic field perpendicular to the plane of the fractal is found to produce gaps in the energy spectrum. A special value of the magnetic flux viz., $\Phi=\Phi_{0} / 2$ is shown to lead to an extreme localization of the electron states as well. The results are corroborated by the density of states calculations, and the valuation two terminal magneto-transport.

\section{Acknowledgments}

Biplab Pal would like to thank DST-INSPIRE Program, India for providing financial assistance through INSPIRE Fellowship (IF110078). Illuminating conversation with Bibhas Bhattacharyya is gratefully acknowledged.
[1] P. W. Anderson, Phys. Rev. 109, 1492 (1958).

[2] E. Abrahams, P. W. Anderson, D. C. Licciardello, and T. V. Ramakrishnan, Phys. Rev. Lett. 42, 673 (1979).
[3] A. MacKinnon and B. Kramer, Phys. Rev. Lett. 49, 695 (1982).

[4] D. J. Thouless, Phys. Rev. Lett. 61, 2141 (1988). 
[5] I. V. Plyushchay, R. A. Römer, and M. Schreiber, Phys. Rev. B 68, 064201 (2003).

[6] A. Rodriguez, L. J. Vasquez, K. Slevin, and R. A. Römer, Phys. Rev. Lett. 105, 046403 (2010).

[7] A. Rodriguez, L. J. Vasquez, K. Slevin, and R. A. Römer, Phys. Rev. B 84, 134209 (2011).

[8] M. Zilly, O. Ujsághy, M. Woelki, and D. E. Wolf, Phys Rev. B. 85, 075110 (2012).

[9] D. M. Jović, M. R. Belić, and C. Denz, Phys. Rev. A 85, 031801 (2012)

[10] K. Y. Bliokh, S. A. Gredeskul, P. Rajan, I. V. Shadrivov, and Y. S. Kivshar, Phys. Rev. B 85, 014205 (2012).

[11] C. Echeverria-Arrondo and E. Ya. Sherman, Phys. Rev. B 85, 085430 (2012).

[12] T. A. Sedrakyan, J. P. Kestner, and S. Das Sarma, Phys. Rev. A 84, 053621 (2012).

[13] S. Aubry and G. André, Ann. Israel Phys. Soc. 3, 133 (1980).

[14] S. Das Sarma, S. He, and X. C. Xie, Phys. Rev. B 41, 5544 (1990).

[15] J. Billy, V. Josse, Z. Zuo, A. Bernard, B. Hambrecht, P. Lugan, D. Clément, L. Sanchez-Palencia, P. Bouyer, and A. Aspect, Nature (London) 453, 891 (2008).

[16] E. E. Edwards, M. Beeler, T. Hong, and S. L. Rolston, Phys. Rev. Lett. 101, 260402 (2008).

[17] G. Roati, C. D'Errico, L. Fallani, M. Fattori, C. Fort, M. Zacanti, G. Modugno, M. Modugno, and M. Inguscio, Nature (London), 453, 895 (2008).

[18] Z. Shi and A. Z. Genack, Phys. Rev. Lett. 108, 043901 (2012).

[19] A. A. Asatryan, L. C. Botten, M. A. Byrne, V. D. Freilikher, S. A. Gredeskul, I. V. Shadrivov, R. C. McPhedran, and Y. S. Kivshar, Phys. Rev. B 85, 045122 (2012).

[20] D. H. Dunlap, H-L. Wu, and P. W. Phillips, Phys. Rev. Lett. 65, 88 (1990); D. H. Dunlap, K. Kundu, and P. W. Phillips, Phys. Rev. B 40, 10999 (1989).

[21] F. A. B. F. de Moura and M. L. Lyra, Phys. Rev. Lett. 81, 3735 (1998).

[22] A. Chakrabarti, S. N. Karmakar, and R. K. Moitra, Phys. Rev. Lett. 74, 1403 (1995).

[23] A. Chakrabarti, S. N. Karmakar, and R. K. Moitra, Phys. Rev. B 50, 13276 (1994).
[24] A. Sánchez, E. Maciá, and F. Dominguez-Adame, Phys. Rev. B 49, 147 (1994)

[25] E. Maciá, Phys. Rev. B 60, 10032 (1999).

[26] E. Maciá, Aperiodic Structures in Condensed Matter, CRC Press (Florida) (2009).

[27] A. Chakrabarti, J. Phys.: Condens. Matt. 8, 10951 (1996).

[28] A. Chakrabarti, Phys. Rev. B 72, 134207 (2005).

[29] W. A. Schwalm and B. J. Moritz, Phys. Rev. B 71, 134207 (2005)

[30] S. Sil, S. K. Maiti and A. Chakrabarti, Phys. Rev. Lett. 101, 076803 (2008).

[31] F. A. B. F. de Moura, R. A. Caetano, and M. L. Lyra, Phys. Rev. B 81, 125104 (2010).

[32] T. Vicsek, Fractal Growth Phenomena (2nd Ed.), World Scientific, Singapore (1992).

[33] R. Rammal and G. Toulouse, Phys. Rev. Lett. 49, 1194 (1982).

[34] E. Domany, S. Alexander, D. Bensimon, and L. P. Kadanoff, Phys. Rev. B 28, 3110 (1983).

[35] Y. Lin, B. Wu, and Z. Zhang, Phys. Rev. E 82, 031140 (2010).

[36] C. S. Jayanthi and S. Y. Wu, Phys. Rev. B 48, 10188 (1993).

[37] S. Sil, S. K. Maiti, and A. Chakrabarti, Phys. Rev. B 79, 193309 (2009).

[38] A. Aharony, Ora Entin-Wohlman, Y. Tokura, and S. Katsumoto, Phys. Rev. B 78, 125328 (2008).

[39] A. Aharony, Ora Entin-Wohlman, Y. Tokura, and S. Katsumoto, Physica E 42, 629 (2010).

[40] J. Vidal, R. Mosseri, and B. Doucot, Phys. Rev. Lett. 81, 5888 (1998).

[41] R. Peierls, Z. Phys. 80, 763 (1933).

[42] B. W. Southern, A. A. Kumar, and J. A. Ashraff, Phys. Rev. B 28, 1785 (1983).

[43] A. Chakrabarti and B. Bhattacharyya, Phys. Rev. B 54, R12625 (1996).

[44] A. Chakraborti, B. Bhattacharyya, and A. Chakrabarti, Phys. Rev. B 61, 7395 (2000)

[45] A. D. Stone, J. D. Joannopoulos, and D. J. Chadi, Phys. Rev. B 24, 5583 (1981) 